\title{
Trial-by-trial alternation of unilateral amygdaloid stimulation
}

\author{
JOHN GAITO \\ York University, Downsview, Ontario, Canada M3J 1P3
}

\begin{abstract}
Previous research indicated that an oscillation effect resulted during sequential alternation of unilateral amygdaloid stimulation with consistent low-latency values for one side and consistent high values for the contralateral one. In this study only one trial of stimulation was applied before alternating to the opposite side. The oscillation tendency resulted under this condition in a similar fashion as in previous studies in which stimulation was continued with one side until six convulsions occurred.
\end{abstract}

The "kindling effect" has been investigated in a number of laboratories as a model of learning, a model of epilepsy, or as an example of behavioral change of interest in its own right (e.g., Gaito \& Gaito, 1974; Goddard, McIntyre, \& Leech, 1969). This event involves a change from normal exploration (Stage 1, NE), to behavioral automatisms (Stage 2, BA; i.e., chewing, eye closure on ipsilateral side, salivation), and finally, to clonic convulsions (Stage 3, CC) in response to electrical stimulation of a specific brain site (e.g., amygdala). In Stage 3 the rat stands on its hindpaws and bilateral convulsions of the forelimbs occur. Behavioral, chemical, electrophysiological, and neurological aspects of this effect have been investigated by many researchers (e.g., Gaito, 1976a).

In recent research in my laboratory with 3 trials/day and stimulation at an intensity of 100 microA for $30 \mathrm{sec}$ using a sequence of alternating unilateral stimulations of the amygdala, usually for 10 alternation phases, an "oscillation effect" was observed (Gaito, $1976 \mathrm{~b})$. The oscillation usually involved low values consistently for the amygdala first stimulated (primary site) and high values consistently for the contralateral amygdala (secondary site). The effect was most prominent in latency data (number of seconds from onset of stimulation to onset of convulsion). The oscillation tendency in the latency measure was remarkably resistant to a number of experimental manipulations (Gaito, Gaito, \& Nobrega, 1977).

The previous experiments were conducted with alternation of stimulation for 10 phases, with a phase consisting of $6 \mathrm{CC}$ from one side before stimulating the opposite side. The present experiments were concerned with stimulation of one side for a single trial before alternating to the opposite side.

\section{EXPERIMENT 1}

\section{Method}

Twelve male Wistar rats (140-190 days of age at the

This research was supported by a grant from the President's National Research Council Fund (York University). beginning of the experiment) had bipolar electrodes inserted in each amygdala. The brain coordinates used for implantation of electrodes into the amygdalae were the same as in many experiments in our laboratory: $.5 \mathrm{~mm}$ posterior to bregma, $4.5 \mathrm{~mm}$ from midline, $8.5 \mathrm{~mm}$ from skull (Gaito, 1976b; Gaito \& Gaito, 1974).

Seven or more days after surgery, brain stimulation began. Each rat was stimulated four times each day ( $1 \mathrm{~h}$ between trials) for $30 \mathrm{sec}$ with a $60-\mathrm{Hz}$ sine wave of 100 microA, using a Lafayette stimulator. Stimulation was alternated from one amygdala to the contralateral one on each trial. Thus, each rat received two stimulations of each amygdala every day. The number of trials to reach the criterion of $6 \mathrm{CC}$ for each side was recorded, as were the latency and duration of the convulsion. On each trial, stimulation terminated after $30 \mathrm{sec}$ (if a convulsion was in progress or if no convulsion had occurred) or at the termination of the convulsion (if the convulsion ceased prior to $30 \mathrm{sec}$ ). This procedure was continued for 10 phases -5 phases for each side. In most cases more phases were achieved for one side than for the other, because the CC stage usually occurred earlier for one side than for the other.

The initial intensity of stimulation was set at 100 microA for all animals; the current was increased in the cases of no CC after 10 trials, until the optimum convulsion intensity was achieved for each animal. In most cases, the CC stage was achieved with 100 microA; with three rats, 420 microA was required on one side; for two others, 560 microA was required.

On the first trial of Phases 3 and 4 , the lowest intensity to elicit a convulsion was determined, and 15 microA was added to handle possible day-by-day fluctuations. This intensity was used in all remaining trials. Because varying amounts of time were required to determine this threshold intensity, the first trial of Phases 3 and 4 were not used in the data analyses.

In each experiment the behavioral designation for the latency and criterion measures was that of primary oscillation (PO), secondary oscillation (SO), or nonoscillation (N). For example, to be classified as an oscillator, the rat had to show a consistent pattern of "low-high" (primary oscillation) or "high-low" (secondary oscillation) in latency and/or criterion data over 8 or 10 of the 10 alternation phases. As in the previous research, duration values were not analyzed.

At the end of the experiment, the animals were sacrificed with an overdose of sodium pentobarbital. Six of the animals were perfused with saline and formalin. The other six were not perfused, so as to contrast the detectability of electrode tracks in nonperfused rats with that from perfused animals. The brains were removed and kept in a $10 \%$ formalin solution for at least 7 days. The brains were then frozen and 50-micron sections were mounted on histological slides. These slides were placed in a photographic enlarger and the negative was projected onto a screen to locate the electrode tips. The enlargement was approximately tenfold. 


\section{Results}

The lack of perfusion did not impair the detectability of electrode tracks; the tips of the electrodes were determined as easily in the nonperfused rats as in those that had been perfused. In the six nonperfused rats, the tips of both electrodes were in the amygdala in five rats. In the sixth rat, one electrode was in the amygdala and the other was in an adjacent structure, the claustrum. Four of the perfused rats had both electrodes in the amygdala; one electrode was in the amygdala and the other was at the junction of the lateral amygdala and the internal capsule in the fifth rat; the sixth rat had one electrode in the amygdala and the other in the ventral part of the internal capsule.

As in previous experiments (Gaito, 1976b, 1978; Nobrega \&' Gaito, 1978), there appeared to be no relationship between type of behavior (PO, SO, NO) and placement of electrode, as long as one or both of the electrodes was in the amygdala or close to the amygdala.

There were 12 nonoscillators in the criterion data in Experiment 1 and four nonoscillators, five primary oscillators, and three secondary oscillators in the latency measure (Table 1). The criterion number of oscillators, 0 , was not significantly different from the number expected (2) with the probability of oscillation set at .17 , using the binomial distribution $(\mathrm{p}>.05){ }^{1}$ However, the eight oscillators in latency data was a significant departure from the number expected $(\mathrm{p}<.05)$.

\section{EXPERIMENT 2}

The trial-by-trial alternation produced the same oscillation patterns in Experiment 1 as had occurred in previous research. Thus, a second experiment was conducted to see if this result would persist.

\section{Method}

One group of 11 rats was treated the same as the animals in Experiment 1 (Group 1). A second set of 12 rats was stimulated in the fashion of the previous research with 4 trials/day, that is, stimulated for $6 \mathrm{CC}$ on the primary site before stimulation of the secondary site, and so on. All rats were approximately 160 days of age at the beginning of the experiment.

No histological analyses were performed on either group because the analyses of previous research (Gaito, 1976b, 1978; Nobrega \& Gaito, 1978) and those of Experiment 1 indicated no relationship between electrode placement and behavioral pattern.

Table 1

Frequency of Different Behavioral Patterns of Rats in Experiments 1 and 2

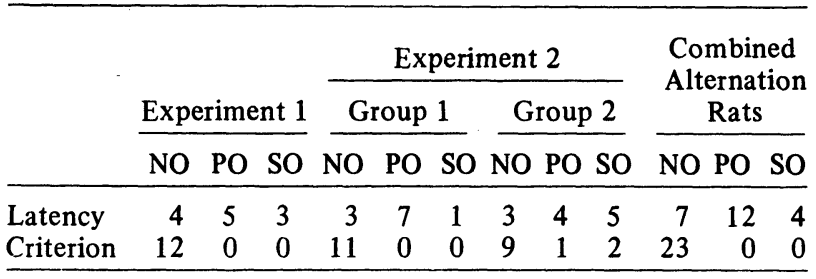

\section{Results}

The results for the trial-by-trial alternation group were essentially the same as in Experiment 1 (Table 1). There were three nonoscillators, seven primary oscillators, and one secondary oscillator in latency data, and 11 nonoscillators in the criterion measure. The finding of eight oscillators (of 11 rats) in latency data was a significant departure from the number expected $(1.9, \mathrm{p}<.05)$. The number of oscillators $(0)$ in criterion data was not a significant result $(p>.05)$.

For Group 2 the finding of nine oscillators (of 12 rats) was a significant result for the latency measure, whereas, the three oscillators observed in criterion data were not significantly different from the number expected (2). These results are consistent with those observed in the previous research.

When the results for Group 1 were combined with those observed in Experiment 1, there were 16 oscillators and seven nonoscillators in latency data and 23 nonoscillators in criterion data. In the former, the number observed is significantly greater than the number expected $(3, \mathrm{p}<.05)$. In the latter measure, the number is significantly less than expected $(p<.05)$; this result rarely occurs and is probably a Type I error.

\section{GENERAL DISCUSSION}

The results of these experiments evaluating the effect of trial-by-trial alternation on the oscillation tendency are similar to the previous research evaluating many experimental variables, especially with the latency measure. The tendency has been prominent in the latency measure, but has been nonexistent, or weak, in the criterion data. The variables evaluated previously include age (young, old) and brain conditions (split-brain rats, intact-brain rats) (Gaito et al., 1977; Nobrega \& Gaito, 1978). Thus, over a wide range of experimental conditions, the oscillation effect has been a robust phenomenon in the latency measure, but a weak or nonexistent event in the criterion measure.

The exact basis for the effect is not known. A number of potential contributors seem to have been excluded. These include chance aspects, differential thresholds for the two sides, differential placement of the two electrodes, differential effectiveness of the two electrodes, and differential natural reactivities of the two sides (Gaito, Nobrega, \& Gaito, in press).

Presumably, there are some inhibitory and/or facilitatory effects from the primary site to the secondary site, and vice versa, to produce the oscillation effect (McIntyre \& Goddard, 1973; Nobrega \& Gaito, 1978). A pattern appears to be set up, either primary oscillation or secondary oscillation, during Phases 1 and 2 , or by Phases 3 and 4 , and most rats continue with the pattern for the remainder of the 10 phases. In one experiment in which rats were stimulated through 50 phases, some rats showed a consistent pattern of oscillation for the 50 phases (Gaito, 1978).

The unilateral sequential alternation procedure and the resulting oscillation effect appear to be useful for obtaining information on some aspects of the events underlying the kindling effect, for example, possible differential electrical synaptic patterns related to the difference in latency values for the primary and secondary sites. Because kindling shows many parallels to learning and to epilepsy and can be considered as a model of consistent behavioral changes in response to an invariant stimulus, experimentation with the oscillation effect may have the potential for providing information relevant to brain function in general. 


\section{REFERENCES}

Garto, J. The kindling effect as a model of epilepsy. Psychological Bulletin, 1976, 83, 1097-1109. (a)

GAITo, J. An oscillation effect during sequential alternations of unilateral amygdaloid stimulations within the kindling paradigm. Physiological Psychology, 1976, 4, 303-306. (b)

Garto, J. The oscillation effect over long-term periods. Bulletin of the Psychonomic Society, 1978, 11, 9-12.

GaIto, J., \& GaIto, S. T. Interanimal negative transfer of the kindling effect. Physiological Psychology, 1974, 2, 379-382.

Gaito, J., Gaito, S. T., \& Nobrega, J. N. A factor analysis of data from ten phases of sequential alternations of amygdaloid stimulation with the kindling paradigm. Physiological Psychology, 1977, 5, 300-310.

Garto, J., Nobrega, J. N., \& Gaito, S. T. Statistical evaluation of several aspects concerning the oscillation effect. Physiological Psychology, in press.

Goddard, G. V., McIntyre, D. G., \& Leech, C. K. A permanent change in brain function resulting from daily electrical stimulation. Experimental Neurology, 1969, 25, 295-330.
MCINTYRe, D. C., \& GodDARD, G. V. Transfer, interference and spontaneous recovery of convulsions kindled from the rat amygdala. Electroencephalography and Clinical Neurophysiology, 1973, 35, 533-543.

Nobrega, J. N., \& GaITo, J. Long term induction of kindled seizures in rats: Interhemispheric factors. The Canadian Journal of Neurological Sciences, 1978, 5, 223-230.

\section{NOTE}

1. The hypothesis that the oscillation patterns are random can be assessed by the one-sample runs test. Using the requirement of 8 or 10 phases of oscillation out of 10 phases would provide 8,9 , or 10 runs. The p of 8,9 , and 10 runs is $.1270, .0317$, and .0079 , respectively; the $p$ of 8,9 , or 10 runs is the sum of these ps, .1666 or .17. Thus, the $p$ that these apparent systematic patterns are random ones would be 17 .

(Received for publication March 14, 1978.) 